\title{
The pre-main-sequence star IP Persei
}

\author{
A. S. Miroshnichenko ${ }^{1,2}$, K. S. Bjorkman ${ }^{1}$, E. L. Chentsov ${ }^{3,4}$, V. G. Klochkova ${ }^{3,4}$, R. O. Gray $^{5}$, \\ P. García-Lario ${ }^{6}$, and J. V. Perea Calderón ${ }^{7}$ \\ 1 Ritter Observatory, Dept. of Physics \& Astronomy, University of Toledo, Toledo, OH 43606-3390, USA \\ 2 Central Astronomical Observatory of the Russian Academy of Sciences at Pulkovo, 196140, Saint-Petersburg, \\ Russia \\ 3 Special Astrophysical Observatory of the Russian Academy of Sciences, Karachai-Cirkassian Republic, \\ Nizhnij Arkhyz, 369167, Russia \\ 4 Issac Newton Institute of Chile, SAO Branch, Russia \\ 5 Dept. of Physics and Astronomy, Appalachian State University, Boone, NC 28608, USA \\ ${ }^{6}$ ISO Data Centre, Astrophysics Division, Space Science Department of ESA, Villafranca del Castillo, \\ Apartado de Correos 50727, 28080 Madrid, Spain \\ 7 INSA SA, Villafranca del Castillo, Apartado de Correos 50727, 28080 Madrid, Spain
}

Received 19 March 2001 / Accepted 21 June 2001

\begin{abstract}
We present the results of high- and low-resolution spectroscopic and broadband multicolour photometric observations of the emission-line A-type star IP Per. Significant variations of the Balmer line profiles and near-IR brightness are detected. Comparison with the spectra of other stars and theoretical models allowed us to derive its fundamental parameters as follows: $T_{\text {eff }} \simeq 8000 \mathrm{~K}, \log g \simeq 4.4, \log L_{\mathrm{bol}} / L_{\odot} \simeq 1.0$. They correspond to the MK type A7 v. We also found that the metallicity of the object's atmosphere is nearly 40 per cent that of the Sun. Our result for the star's gravity implies that it is located at the zero-age main-sequence. We conclude that IP Per is a pre-main-sequence Herbig Ae star, and belongs to the group of UX Ori-type stars showing irregular photometric minima. A recent result by Kovalchuk \& Pugach (1997), that IP Per is an evolved high-luminosity star, is not confirmed. The discrepancy in the $\log g$ determination, which led to the difference in the luminosity, seems to be due to uncertainties in the échelle data reduction for broad lines and a different estimate for the star's temperature.
\end{abstract}

Key words. stars: emission-line - stars: pre-main-sequence - stars: individual IP Per - techniques: spectroscopic, techniques photometric

\section{Introduction}

Herbig Ae/Be stars are pre-main-sequence intermediatemass $\left(2-10 M_{\odot}\right)$ objects which were discovered about 40 years ago (Herbig 1960). They usually show variable brightnesses, polarization, and emission-line spectra which imply the presence of a significant amount of circumstellar matter around these stars. Earlier studies of their physical parameters suggested that they are similar to those of main-sequence stars (Strom et al. 1972) except for the nuclear energy source, which is thought to be deuterium burning (Palla \& Stahler 1993). High-resolution spectroscopic studies of Herbig Ae/Be stars are usually focused on emission lines (Böhm \& Catala 1994) or investigation

Send offprint requests to: A. S. Miroshnichenko, e-mail: anatoly@physics.utoledo.edu of the line variations (Böhm \& Catala 1995). However, such issues as precise measurements of these stars' photospheric parameters and chemical abundances have not been systematically approached. The lack of such data hampers more detailed studies of stellar evolution in the vicinity of the zero-age main-sequence and makes uncertain the stellar age determinations, which are important for problems such as the onset of planet formation and evolution of circumstellar matter. A method for precise spectral classification of pre-main-sequence Atype stars on the basis of low-resolution spectroscopy has been recently developed by Gray \& Corbally (1998), who found a few metal-deficient objects. In order to measure their fundamental parameters and metallicity with a high accuracy, high-resolution spectroscopy in combination with photometry is needed. In this paper we report an 
application of this method. We present our recent multiwavelength photometric and various spectroscopic observations and investigate the properties of a poorlystudied Herbig Ae star IP Per. Similar studies of other Herbig Ae/Be stars will be reported in a series of forthcoming papers.

IP Per was discovered as a variable star of the Algol type by Hoffmeister (1949). The possible eclipsing nature of its variations was supported by Kardopolov \& Phylipjev (1982). These authors detected two nearly 10day long minima and deduced a 1.94672-day period on the basis of $53 B V R$ observations, which were obtained within 2 years. However, studies by Meinunger (1967) and Wenzel (1978), based on longer-term data, showed that this variability is irregular. From his $U B V$ photometric data, Wenzel (1978) estimated the spectral type of IP Per as A3. Glass \& Penston (1974) detected a strong nearIR excess characteristic of circumstellar dust radiation. This finding was supported by the IRAS detection of a longer-wavelength excess radiation (e.g. Weintraub 1990). Herbig \& Bell (1988) included IP Per in their catalogue of emission-line stars of the Orion population. Finally, Thé et al. (1994) listed the object as a pre-main-sequence intermediate-mass star in their catalogue of members and candidate members of the Herbig Ae/Be stellar group.

The star's photometric variations are similar to those of Algol-type Herbig Ae stars (Grinin et al. 1991), which display irregular short-term brightness minima accompanied by an increase of the optical reddening. This subgroup of Herbig Ae stars is also called the UX Oritype stars, or UXOrs. Natta et al. (1997) argued that UXOrs are typical pre-main-sequence intermediate-mass stars, and their variability is due to a low inclination of the equatorial plane of an aspheric circumstellar nebula rather than to evolutionary effects.

Pirzkal et al. (1997) detected no nearby companion in the $K$-band down to an angular distance of 0 ". 4 from IP Per, while Testi et al. (1997) found no clustering around the star. The latter result is common for premain-sequence stars with spectral types later than B7 and shows that such stars are formed in small protostellar complexes. Two other Herbig Ae stars, XY Per (Thé et al. 1994) and GSC 1811-0767 (Miroshnichenko et al. 1999a), are located close to the position of IP Per. Thus, IP Per is not completely isolated like some other UXOrs (e.g. UX Ori). Gray \& Corbally (1998) classified IP Per as an A7-type star on the basis of classification-resolution $(3.6 \AA$ per 2 pixels) spectroscopy and noticed that the Ca II $\mathrm{K}$ line and the general metallic-line spectrum are slightly weak. These authors suggest that IP Per is an example of stars in which accretion of metal-depleted gas producies a slightly metal-weak nature.

Although the properties of IP Per closely resemble those of Herbig Ae stars, there is another opinion on its evolutionary state as well as on that of the whole UXOrs group. Kovalchuk \& Pugach (1997) obtained mediumresolution (resolving power $R \sim 6000$ ) spectroscopic observations of 19 UXOrs, and fitted their Balmer line
Table 1. Optical photometry of IP Per.

\begin{tabular}{rccccc}
\hline JD 2450000+ & $V$ & $U-B$ & $B-V$ & $V-R$ & $V-I$ \\
\hline 420.22 & 10.43 & 0.11 & 0.32 & 0.30 & 0.56 \\
422.24 & 10.38 & 0.16 & 0.34 & 0.30 & 0.63 \\
424.16 & 10.56 & 0.14 & 0.34 & 0.34 & 0.52 \\
425.23 & 10.54 & 0.08 & 0.30 & 0.35 & 0.62 \\
427.21 & 10.48 & 0.17 & 0.29 & 0.35 & 0.59 \\
428.21 & 10.49 & 0.15 & 0.30 & 0.35 & 0.61 \\
429.25 & 10.51 & 0.21 & 0.35 & 0.35 & 0.62 \\
439.19 & 10.36 & 0.09 & 0.31 & - & - \\
1154.20 & 10.47 & 0.09 & 0.34 & 0.33 & 0.55 \\
1156.13 & 10.47 & - & 0.31 & - & - \\
1156.20 & 10.47 & 0.23 & 0.31 & 0.34 & 0.63 \\
1156.23 & 10.48 & - & 0.37 & - & - \\
1156.24 & 10.46 & - & 0.36 & - & - \\
1156.27 & 10.49 & - & 0.34 & - & - \\
1156.29 & 10.49 & - & 0.34 & - & - \\
1157.20 & 10.49 & 0.18 & 0.32 & 0.33 & 0.61 \\
1159.21 & 10.47 & 0.20 & 0.34 & 0.33 & 0.59 \\
1160.23 & 10.46 & 0.17 & 0.31 & 0.34 & 0.59 \\
1161.17 & 10.50 & 0.18 & 0.31 & 0.37 & 0.66 \\
\hline & & & & &
\end{tabular}

profiles $(\mathrm{H} \beta$ through $\mathrm{H} \delta$ ) to the theoretical ones from the Kurucz (1979) model atmospheres. The results indicate that most of the objects have significantly lower gravities (log $g \sim 2-3$ ) than those expected for main-sequence stars of similar spectral types. Kovalchuk \& Pugach (1997) concluded that this small group of Herbig Ae stars contains evolved rather than young stars. For IP Per they derived the following fundamental parameters: $T_{\text {eff }}=8800 \mathrm{~K}$, $\log g=2.0$.

In order to investigate the properties of IP Per in more detail and to address the above problems, we obtained a number of different observations of the star in 1996-2000. Here we summarize the data accumulated so far (including those from the literature), refine the star's fundamental parameters, and discuss its evolutionary state.

\section{Observations}

The photometric UBVRI observations of IP Per were obtained in November-December 1996 and in December 1998 at a 1-meter telescope of the Tien-Shan Observatory (Kazakhstan) with a two-channel photometer-polarimeter (Bergner et al. 1988). The errors of individual observations, which are presented in Table 1, do not exceed $0.02 \mathrm{mag}$. HD 22418 was used as a comparison star, while the instrumental system stability was controlled using other standard stars observed during each night. Six observations (of which 5 were in the $B$ and $V$ bands only) were taken during 4 hours on 1998 December 8 to search for short-term variations.

On 1999 September 17 and on 2000 July 25 we obtained $J H K$ photometry of the star at the $1.55-\mathrm{m}$ Carlos 
Table 2. Near-IR photometry of IP Per.

\begin{tabular}{lcccccc}
\hline JD 2450000+ & $J$ & $H$ & $K$ & $L$ & $M$ & Ref. \\
\hline & - & 8.23 & 7.47 & 6.61 & - & a \\
1092 & $9.13 \pm 0.02$ & $8.39 \pm 0.02$ & $7.57 \pm 0.01$ & - & - & b \\
1439.10 & $9.11 \pm 0.04$ & $8.29 \pm 0.02$ & $7.39 \pm 0.01$ & - & - & CST \\
1600.23 & - & - & $7.73 \pm 0.04$ & $6.56 \pm 0.05$ & $6.11 \pm 0.05$ & IRTF \\
1751.75 & $9.23 \pm 0.03$ & $8.56 \pm 0.03$ & $7.77 \pm 0.03$ & - & - & CST \\
\hline
\end{tabular}

a Data from Glass \& Penston (1974).

a Data from the 2MASS survey (Skrutskie et al. 1997).

Sánchez Telescope (CST), operated by the Instituto de Astrofísica de Canarias at the Spanish Observatorio del Teide (Tenerife, Spain). We used a CVF infrared spectrophotometer equipped with an InSb photovoltaic detector, operating at the temperature of liquid nitrogen, with a photometric aperture of $15^{\prime \prime}$ and a chopper throw of $30^{\prime \prime}$ in the E-W direction to subtract the contribution from the background sky. The star was observed $4-5$ times in each band. The Teide photometric system is described in Arribas \& Martínez-Roger (1987), as well as its relations with other standard photometric systems.

On 2000 February 26 we obtained KLM photometry of IP Per at the 3-m NASA IRTF, equipped with a singleelement gallium-doped germanium bolometer, at Mauna Kea (Hawaii, USA). The photometric aperture of $10^{\prime \prime}$ and an $11 \mathrm{~Hz}$ chopper throw of $15^{\prime \prime}$ in the N-S direction was used. Four 20-second exposures were taken in each band. The magnitudes for HR 1457 ( $M$-band only) and HR 1641, taken from the IRTF standard stars list, were used for calibration. The averaged results of our near-IR photometry are presented in Table 2 .

The high-resolution spectrum of IP Per was obtained on 1999 January 7 at the 6 -m telescope of the Special Astrophysical Observatory (SAO) of the Russian Academy of Sciences with the échelle-spectrometer PFES (Panchuk et al. 1998) and a $1140 \times 1170$ pixel CCD detector. The spectral range was $4577-7820 \AA$ with the mean $R \sim 15000$.

Five classification-resolution (3.6 $\AA$ per 2 pixels) spectra of IP Per were obtained using the Gray/Miller Cassegrain spectrograph on the $0.8-\mathrm{m}$ telescope of the Dark Sky Observatory of the Appalachian State University. These spectra were obtained with a 600 line $\mathrm{mm}^{-1}$ grating in the first order using a $1024 \times 1024$ Tektronics thinned, back-illuminated CCD. The spectral range is $3800-5600 \AA$. The spectra were reduced using standard methods under $\mathrm{IRAF}^{1}$, and have signal-to-noise ratios exceeding 100.

\footnotetext{
${ }^{1}$ IRAF is distributed by the National Optical Astronomy Observatories, which are operated by the Association of Universities for Research in Astronomy, Inc., under contract with the National Science Foundation.
}

High-resolution spectra of several bright A5-F0 stars with known fundamental parameters (HR 2852, HR 3569, and HR 5435) were obtained with a fiber-fed échelle spectrograph and a Wright Instruments Ltd. CCD camera at the 1-meter telescope of Ritter Observatory for comparison purposes. The spectra, from 5285 to $6597 \AA$, consisted of nine non-overlapping $\simeq 70 \AA$ wide orders, with spectral resolving power $R \simeq 26000$. The data were reduced with IRAF. Additionally, a spectrum of HR $4738(R \simeq 25000, \lambda \lambda$ 5160-6600 $\AA$ ) was taken with the CCD equipped échelle-spectrometer LYNX (Panchuk et al. 1999) mounted at the Nasmyth focus of the 6-meter SAO telescope.

\section{Results}

\subsection{Light curve and spectral energy distribution}

Figure 1a shows the photoelectric $V$-band light curve of IP Per between 1973 and 1998 compiled from the data of Kardopolov \& Phylipjev (1982), Pugach (1996), Eimontas \& Sudžius (1998), and this work. At least 5 minima can be recognized during this period. The light curve is typical for an UX Ori-type star with a duration for the minima from 10 to 50 days (Wenzel 1978). The data presented in Fig. 1 are suggestive of a marginal increase of the star's activity, since only 3 minima were detected during the period 1956-1977 (Wenzel 1978). Previously other periods of increased activity were observed in 19361940 and 1949-1955. No significant short-term variations $(\Delta V \leq 0.02 \mathrm{mag})$ were detected during our 4-hour observations on 1998 December 8, when a possible eclipse was expected from the ephemeris of Kardopolov \& Phylipjev (1982).

Colour-indices for typical UXOrs become larger as the star goes into a minimum; however, this is not obviously seen in our data for IP Per (Fig. 1b). At the same time, this is a feature of deep minima, which are thought to be due to occultations of the stellar disk by dusty clouds from the circumstellar envelope (e.g., Grinin et al. 1991), while small-amplitude brightness variations near the brightest state may be caused by a different mechanism (e.g., variations in the gaseous part of the envelope) and may be accompanied by different color changes. Furthermore, the 

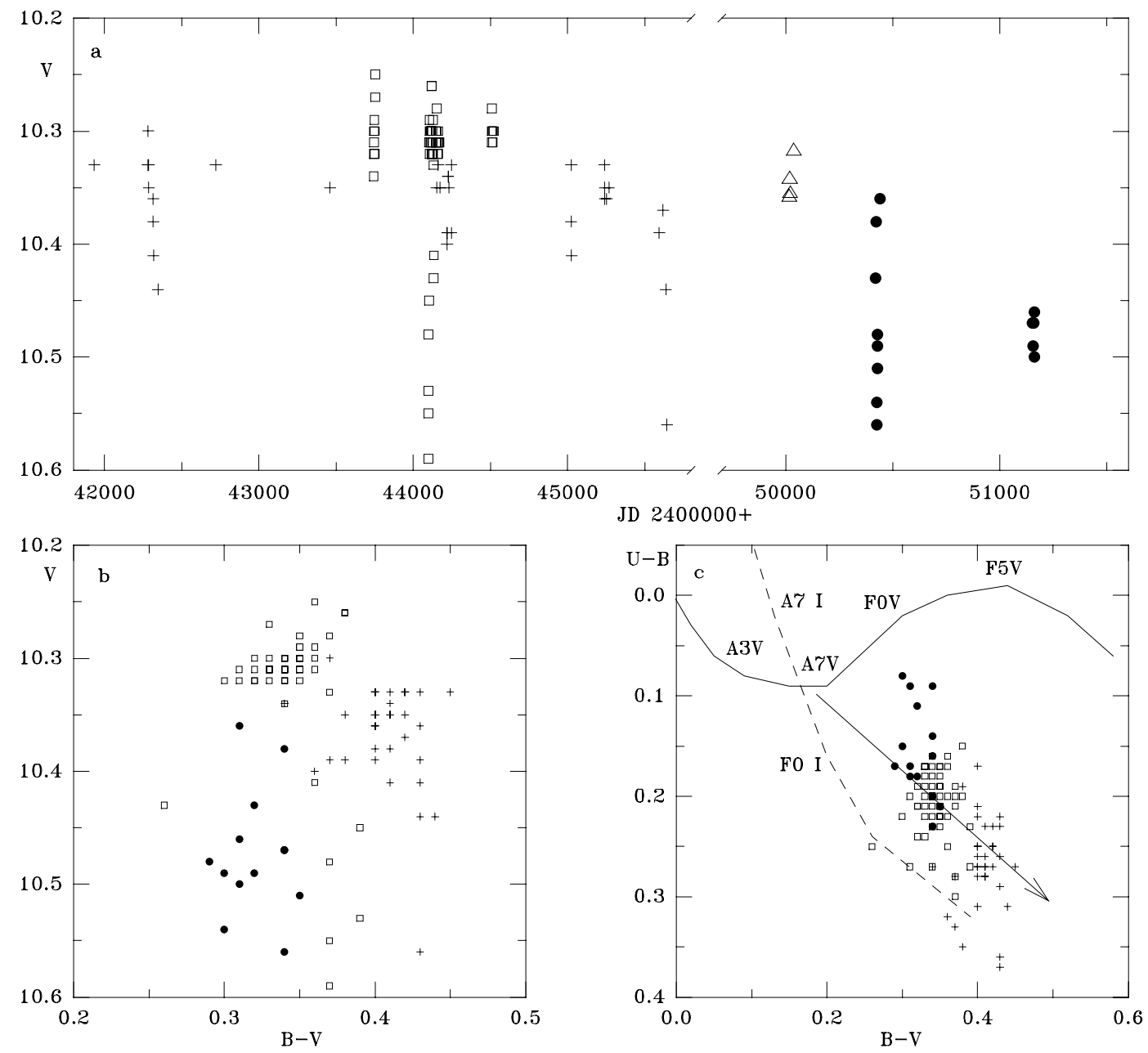

Fig. 1. Photometric variations of IP Per. a) The $V$-band light curve. b) Colour-magnitude diagramme. c) Colour-colour diagramme. The solid line represents intrinsic colour-indices of dwarfs (Straizhis 1977), while the dashed line those of supergiants. Data from Pugach (1996) are denoted by pluses, from Kardopolov \& Phylipjev (1982) by open squares, from Eimontas \& Sužius (1998) by open triangles, and from this paper by filled circles. The line with an arrow shows the interstellar extinction vector.

scattering of the data obtained by different authors may be in part due to different comparison stars and different instrumental systems used. However, a similar scattering exists in the Wenzel (1978) data, unpublished in numerical form.

The trend in the $U-B$ vs. $B-V$ plane (Fig. 1c) is close to the interstellar reddening vector with slope $\left(E_{U-B} / E_{B-V}=0.72 \pm 0.03\right)$ measured using photometric data for stars in the $2^{\circ} \times 2^{\circ}$ region centered on the position of IP Per. This vector, applied to the star's colour-indices for de-reddening, suggests a mean spectral type A6 V or A8 I. Irrespective of the luminosity type chosen, the interstellar colour-excess is found to be $E_{B-V} \simeq 0.15 \mathrm{mag}$ assuming no circumstellar contribution to the star's $B-V$ in the maximum brightness.

The spectral energy distribution (SED) between 0.3 and $100 \mu \mathrm{m}$ for the maximum optical and near-IR brightness is shown in Fig. 2. The IRAS fluxes were taken from a paper by Weaver \& Jones (1992), who corrected the original Point Source Catalog measurements using the
ADDSCAN technique. The near-IR measurements collected in Table 2 show a variable brightness in the JHK bands, whose amplitude increases towards longer wavelengths $(\Delta J \sim 0.1 \mathrm{mag}, \Delta H \sim 0.3 \mathrm{mag}, \Delta K \sim 0.4 \mathrm{mag})$. Such a behaviour is not likely to be due to an Algoltype minimum, which usually affects the SED shortward of $2 \mu \mathrm{m}$ and produces a fading decreasing with wavelength (Kolotilov et al. 1977), but rather suggests that there may be another component of the variability in the near-IR region. Future photometric observations, especially quasi-simultaneous in the optical and near-IR regions, are needed to investigate this effect.

A large IR-excess with two peaks is clearly seen longward of $1 \mu \mathrm{m}$. Potentially, the shape of the IR-excess can be explained by a combination of an optically-thin spherical shell and an optically-thick circumstellar disk as it was shown by Miroshnichenko et al. (1999c) for the A8e premain-sequence star MWC 758 (HD 36112). Unfortunately, the disk contribution cannot be well constrained, because no flux measurements have been obtained longward of 


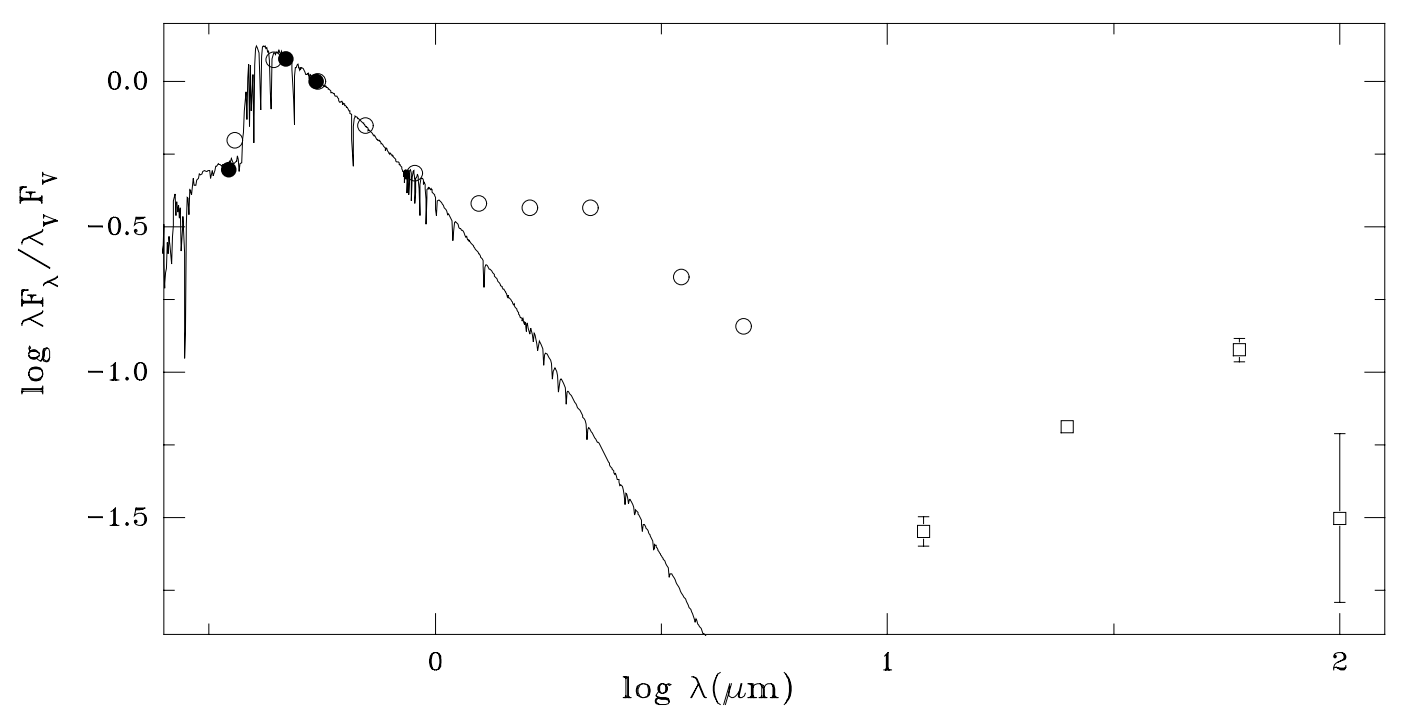

Fig. 2. The SED of IP Per. The solid line represents the synthetic spectrum for $T_{\text {eff }}=7960 \mathrm{~K}$ and $\log g=4.38$. The Strömgren $u, b$ and $y$ band fluxes are shown by filled circles, the Johnson UBRIJHKLM band fluxes by open circles, while the IRAS data by open squares. A reddening of $E_{b-y}=0.11 \mathrm{mag}$ and $V=y=10.34 \mathrm{mag}$ was assumed.
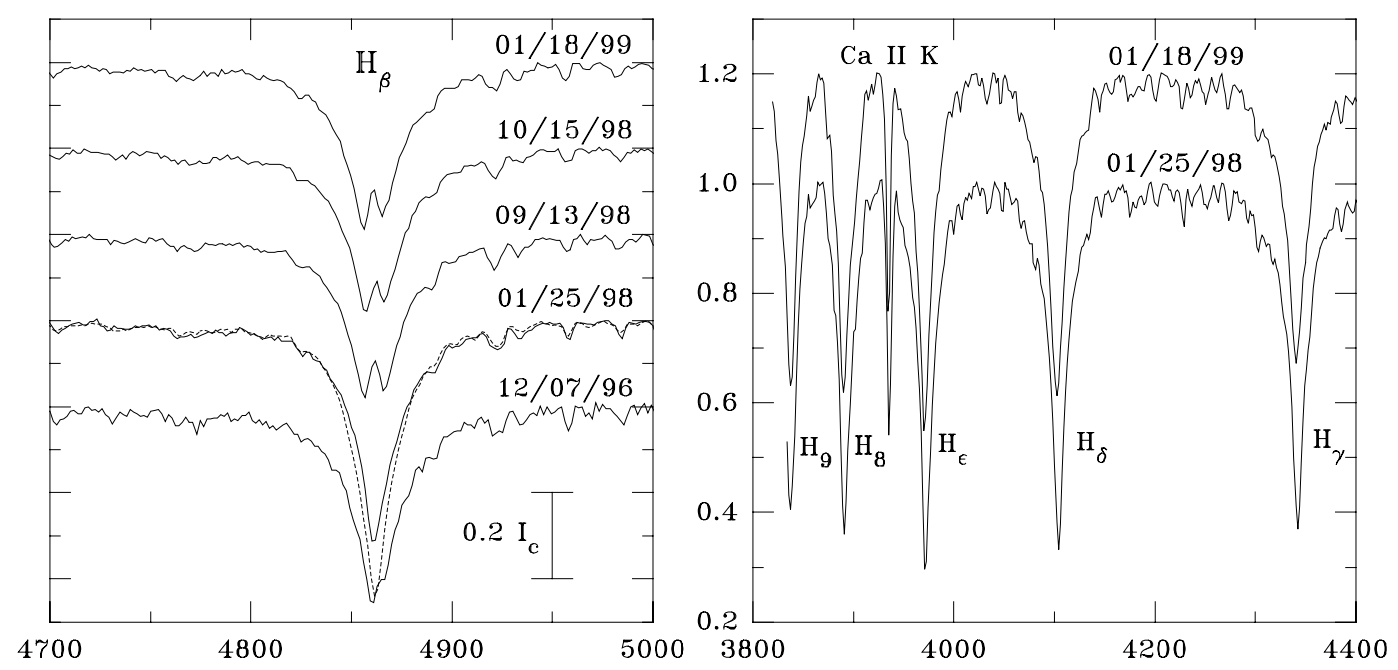

Fig. 3. The DSO spectra of IP Per (solid lines). The spectra are labelled by the dates they were taken. Left panel shows the $\mathrm{H} \beta$ line variations. The dashed line represents the DSO spectrum of HD 32509 from Miroshnichenko et al. (1999c). Right panel shows the variations in other Balmer line profiles by comparison of the spectrum with a high and a low emission level in $\mathrm{H} \beta$.

$100 \mu \mathrm{m}$, where the disks' outer parts dominate the emergent radiation. Additionally, the second peak strength may be affected by the IR cirrus emission at 60 and $100 \mu \mathrm{m}$ (Ivezić \& Elitzur 1995).

\subsection{Spectrum}

The SAO spectrum of IP Per contains a large number of absorption lines and very few emission features. Most of the absorptions are due to neutral metals, while singly ionized species are also present. The emission is seen only in the Balmer lines $(\mathrm{H} \alpha$ and $\mathrm{H} \beta)$ and the $\mathrm{He} \mathrm{I} 5876 \AA$ line. The forbidden neutral oxygen lines at 5577 and $6300 \AA$ observed in emission are presumably telluric. Several diffuse interstellar absorption bands (DIBs) seen at 5780, 6278, and $6614 \AA$ are weak, but they suggest a certain amount of interstellar reddening in the line of sight. The list of lines identified in the spectrum of IP Per with the help of a catalogue by Coluzzi (1993) is presented in Appendix.

The DSO spectra show a variable emission component in the $\mathrm{H} \beta$ line which correlates with the strengths of the higher members of the series (Fig. 3). No noticeable variations are seen in the $\mathrm{Ca}$ II $\mathrm{K}$ line. Although almost no signs of $\mathrm{H} \beta$ emission are present in the spectrum of 1998 January 25, all the other DSO spectra show significant emission at $\mathrm{H} \beta$. Other features in the spectrum show mild variability. In particular, the metallic-line spectrum was slightly stronger on 1996 December 7 when the star was close to photometric minimum. Overall, the star displays detectable spectral variations, but they are not dramatic. 


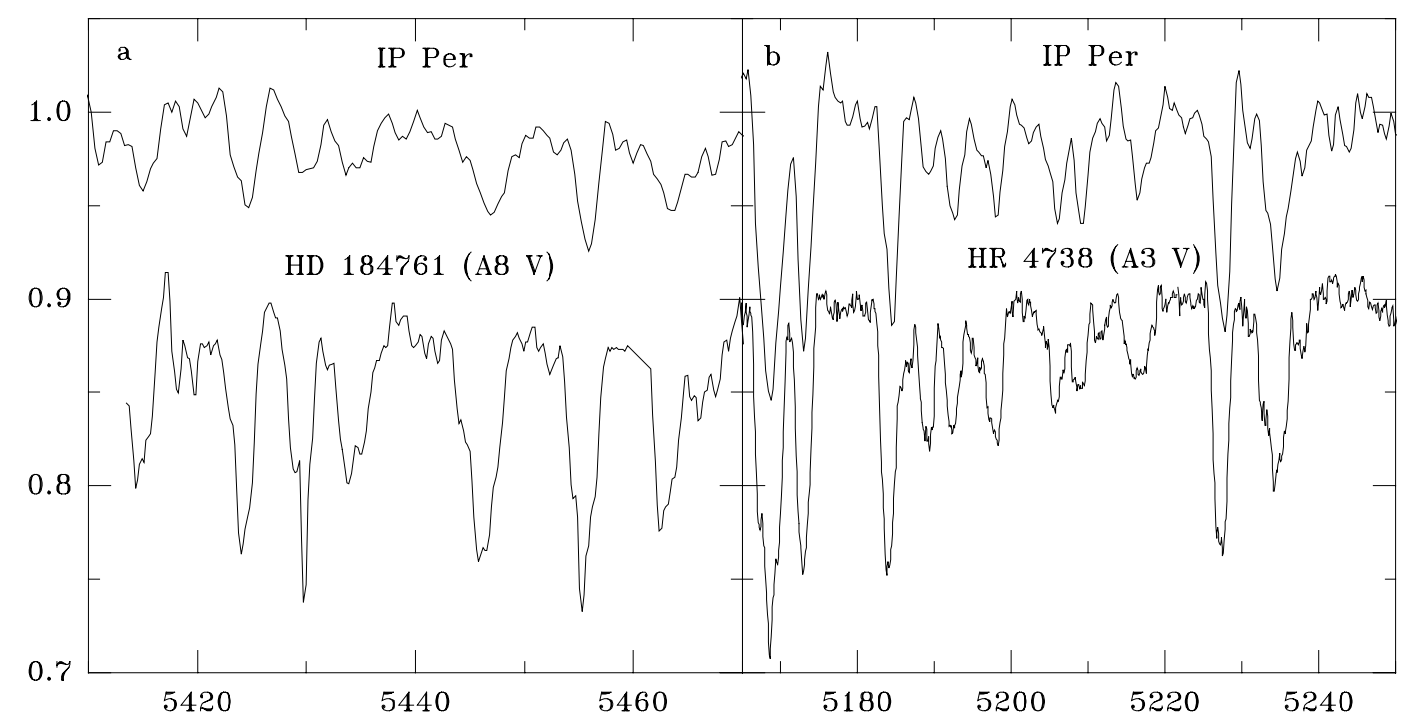

Fig. 4. Comparison of the metallic line spectrum. a. IP Per and HD 184761; b. IP Per and HR 4738. The wavelength scale is given in $\AA$.

Table 3. Classification of the DSO spectra of IP Per.

\begin{tabular}{lrl}
\hline \multicolumn{1}{l}{ Date } & JD 2450000+ & Spectral type \\
\hline 1996 December 07/08 & 425.668 & A7 kA3 mA4 III:er \\
1998 January 25/26 & 839.592 & A7 kA3 mA3 III:e \\
1998 September 13/14 & 1070.830 & A7 kA2.5 mA3 III:e(r) Bd < \\
1998 October 15/16 & 1102.771 & A7 kA2.5 mA3 III:e(r) Bd < \\
1999 January 18/19 & 1197.557 & A7 KA2.5 mA3 III:e(r) Bd < \\
\hline
\end{tabular}

These spectra have been classified on the extension of the MK classification system devised by Gray \& Corbally (1998) for classifying Herbig Ae stars. The classifications are presented in Table 3. In summary, these spectral types imply that IP Per is an A7 star, with an uncertain luminosity class (possibly a giant, although a dwarf luminosity class cannot be excluded) and appears to be slightly metal weak (see discussion in Gray \& Corbally 1998). The hydrogen lines indicate that $T_{\text {eff }}$ is that of an A7 star, but the metallic lines (including the Ca II K-line) have the strength of an A3 star.

A quick look at the high-resolution spectrum allows a rough estimate of the star's fundamental parameters. The presence of noticeable neutral metallic lines in the region 5000-5500 A suggests a temperature type cooler than A3. The equivalent width of the O I triplet at $7772-7775 \AA$, which is used as a luminosity indicator (e.g. Faraggiana et al. 1988), is $0.45 \AA$ in our spectrum of IP Per, suggesting a main-sequence luminosity.

To derive the fundamental parameters with a better accuracy, we made a detailed comparison of the IP Per spectrum with those of other stars and with theoretical spectra, which were computed with the program SPECTRUM (Gray \& Corbally 1994) using the ATLAs9 models of Kurucz (1993). The width of the majority of unblended lines is consistent with a rotational velocity of $v \sin i=70 \pm 20 \mathrm{~km} \mathrm{~s}^{-1}$. This estimate was derived on the basis of the comparison with the line spectra of HR $2852\left(\mathrm{~F} 0 \mathrm{~V}, v \sin i=63 \mathrm{~km} \mathrm{~s}^{-1}\right.$ ) and HR 4738 (A3 IV, $\left.v \sin i=75 \pm 10 \mathrm{~km} \mathrm{~s}^{-1}\right)$. Another source of this estimate is the comparison with the theoretical profiles broadened rotationally and convolved with a $0.4 \AA$ full-width at half maximum Gaussian, which is approximately the resolution of the SAO spectrum of IP Per. These parameters were used in the calculations of all the theoretical spectra.

\subsubsection{Modelling}

The metallic line strength in A-type stars increases, as the $T_{\text {eff }}$ goes down. They also depend on metal abundances, so that metal deficient stars have weaker metallic lines than those with normal (solar) abundances. In order to separate these two effects, both spectroscopic and photometric parameters have to be taken into account. The complexity of this problem is illustrated in Fig. 4, where parts of our high-resolution spectrum of IP Per are compared to those of other A-type stars with different $T_{\text {eff }}$. The metallic line strengths are close to those of HR $4738\left(T_{\text {eff }}=8300 \mathrm{~K}\right.$, A3 IV, Gray \& Garrison 1989), while they are noticeably weaker than those of HD $184761\left(T_{\text {eff }}=7500 \mathrm{~K}\right.$, A8 v, Miroshnichenko et al. 1999c). Both these comparison stars presumably have metallicity close to solar. At the same time, the photometric data and our DSO spectra suggest that IP Per is an A7 star.

This diagnosis may be confirmed by spectral synthesis. One of us (ROG) has devised a technique (see Gray et al. 2001, in preparation, for details) that utilizes a multidimensional downhill simplex method to fit the basic parameters of a star, i.e. the effective temperature, the gravity, the microturbulent velocity $\left(\xi_{t}\right)$ and the metallicity $([M / H])$ by iteratively choosing the model that best 
matches the observed classification spectrum and fluxes from Strömgren uvby photometry. The synthetic spectra used in this technique were computed with the program SPECTRUM using the ATLAS9 models of Kurucz (1993).

Unfortunately, Strömgren uvby photometry is not available for IP Per, but Eimontas \& Sudžius (1998) have observed this star using intermediate-band Vilnius photometry. It turns out (cf. Straižys et al. 1996) that magnitudes in the Vilnius $U, Y$ and $V$ bands are related linearly to the Strömgren $u, b$ and $y$ bands respectively and may be satisfactorily transformed once one finds the respective zero-point differences. We have determined these zero-point differences from Vilnius standard stars, which also have Strömgren photometry. This yields the following magnitudes in the Strömgren system: $u=12.14 \mathrm{mag}$, $b=10.58 \mathrm{mag}, y=10.34 \mathrm{mag}$. These magnitudes can be converted to fluxes using the formulae of Gray (1998). The $v$-band flux is not used in the SIMPLEx method because the $\mathrm{H} \delta$ line falls in the middle of this band. A reddening of $E_{b-y}=0.11 \mathrm{mag}$ which corresponds to $E_{B-V}=0.15 \mathrm{mag}$, which was adopted elsewhere in this paper, was used.

The SIMPLEX method yields, as a mean for the five classification spectra, the following fundamental parameters: $T_{\text {eff }}=7960 \pm 50 \mathrm{~K}, \log g=4.38 \pm 0.04, \xi_{t}=2.0 \pm 0.5 \mathrm{~km} \mathrm{~s}^{-1}$ and $[M / H]=-0.41 \pm 0.04$. The quoted errors are formal standard deviations and are probably over optimistic by a factor of two as independent photometry was not available for each of the five spectra.

The fit (synthetic emergent spectrum calculated with the above fundamental parameters) with both the observed spectra and the fluxes from Strömgren $u b y$ photometry are satisfactory, as can be seen from Figs. 2 and 7. In Fig. 2 we also compare the fit with the de-reddened fluxes from Johnson UBVRI photometry at maximum brightness of the star. The Johnson system bandpasses are much broader than those of the Strömgren system and include strong Balmer lines (the $B$ and $R$ bands) and the Balmer jump ( $U$-band). This results in deviations of the observed fluxes from the monochromatic fluxes of the fit. Nevertheless, the deviations can be explained by only this effect for the $U B V R$-fluxes, while the $I$-band flux may contain a contribution of the circumstellar radiation. Thus, the SIMPLEX fit largely confirms the spectral types in Table 3 ; the $T_{\text {eff }}$ agrees well with the A7 temperature type, and the star does appear to be significantly metal-weak. However, the gravity $(\log g=4.38)$ is more characteristic of a dwarf than a giant.

\subsubsection{Balmer lines}

Both Balmer lines recorded in our SAO spectrum display emission components. However, they show broad wings, which allow comparison with those of normal stars. The slope of the wings of the Balmer lines in the region between 7500 and $9000 \mathrm{~K}$ weakly depend on $T_{\text {eff }}$. Indeed, the $\mathrm{H} \alpha$ wings of $\mathrm{HD} 184761$ ( $T_{\mathrm{eff}}=7500 \mathrm{~K}$, $\log g \simeq 4.3$, Miroshnichenko et al. 1999c), HR 3569 (A7 V,
$T_{\text {eff }}=8150 \mathrm{~K}, \log g=4.28 \pm 0.05$, Smalley \& Dworetsky 1993), and HR 5435 (A7 IV, $T_{\text {eff }}=7860 \mathrm{~K}, \log g \simeq 3.9$, Gubotchkin \& Miroshnichenko 1991) almost coincide with those of IP Per (Fig. 5a). The scatter of these stars' gravities suggests that fitting the $\mathrm{H} \alpha$ wings is capable of providing an accuracy of $\sim 0.2$ in $\log g$.

The shape of the broad Balmer line profiles, such as $\mathrm{H} \alpha$ and $\mathrm{H} \beta$, in our high-resolution spectrum is a bit uncertain because of a number of obstacles, which make the continuum determination (and, hence, the whole profile shape) difficult. For example, the $\mathrm{H} \beta$ line was observed at edges of two échelle orders in our SAO spectrum. Furthermore, the PFES échelle order widths $(120 \AA$ near $\mathrm{H} \beta$ and $170 \AA$ near $\mathrm{H} \alpha$ ) are comparable with the broad photospheric line width of A-type stars. Curvature of the échelle orders may insert an uncertainty in the wing's slope determination. Another potential source of concern could be a different level of scattered light inside different spectrographs which might affect absorption lines depths. In all the spectrographs used this level is small $(\leq 2 \%)$ and would affect only deep lines, none of which are detected in the spectrum of IP Per. Because of the above mentioned problems, we compare IP Per with normal stars only qualitatively, making our conclusions about the star's fundamental parameters from the modelling (see Sect. 3.2.1).

On the other hand, the low-resolution DSO spectra show that the Balmer line wings of IP Per are close to those of HR 1412, an A7 III standard star with $\log g=3.5$ (see also Gray \& Corbally 1998). The $\log g$ value for HR 1412 was calculated using the HIPPARCOS distance ( $D=45.7 \pm 1.2 \mathrm{pc}, \mathrm{ESA} 1997)$, the absolute magnitude $\left(M_{\mathrm{bol}}=0.1 \mathrm{mag}\right)$, the mass $\left(2.37 M_{\odot}\right.$, Lastennet et al. 1999), and $T_{\text {eff }}=8000 \mathrm{~K}$ (Solano \& Fernley 1997). As it is shown in Fig. 6, the DSO spectra of IP Per can be fitted well with the synthetic spectrum for $T_{\text {eff }}=7960 \mathrm{~K}$ and $\log g=4.38$. Furthermore, the Balmer line wings and most of the metallic lines in the DSO spectra of IP Per nearly coincide with those of another young star, HD 32509 (A5 V), whose spectrum was obtained with the same spectrograph (Miroshnichenko et al. 1999c). HD 32509 shows no Balmer line emission and has $\log g=4.1 \pm 0.4$, which was estimated on the basis of the fundamental parameters derived by Miroshnichenko et al. (1999c). When emission is clearly present in the $\mathrm{H} \beta$ line of IP Per, its other Balmer line strengths are noticeably lower than those in HD 32509. However, when the emission decreases, the higher Balmer lines look very similar in the two objects (Fig. 3). The remaining difference is probably due to a lower rotational velocity of IP Per (120 $\mathrm{km} \mathrm{s}^{-1}$ for HD 32509). Finally, our synthetic spectrum for $T_{\text {eff }}=7960 \mathrm{~K}$ and $\log g=4.38$ shows a good agreement with the $\mathrm{H} \alpha$ and $\mathrm{H} \beta$ wings of IP Per observed at high-resolution (see Figs. 5b and 7e). It is also seen in Fig. $5 \mathrm{~b}$ that the wing slope of the $\mathrm{H} \alpha$ line of a supergiant HD 59612 (A5 Ib, $T_{\text {eff }}=8500 \mathrm{~K}$, $\log g=1.5$, Verdugo et al. 1999) is noticeably different from those of IP Per and our model spectrum.

As we mentioned above (see Sect. 1), Kovalchuk \& Pugach (1997) fitted the $\mathrm{H} \gamma$ and $\mathrm{H} \delta$ profiles of IP Per, 

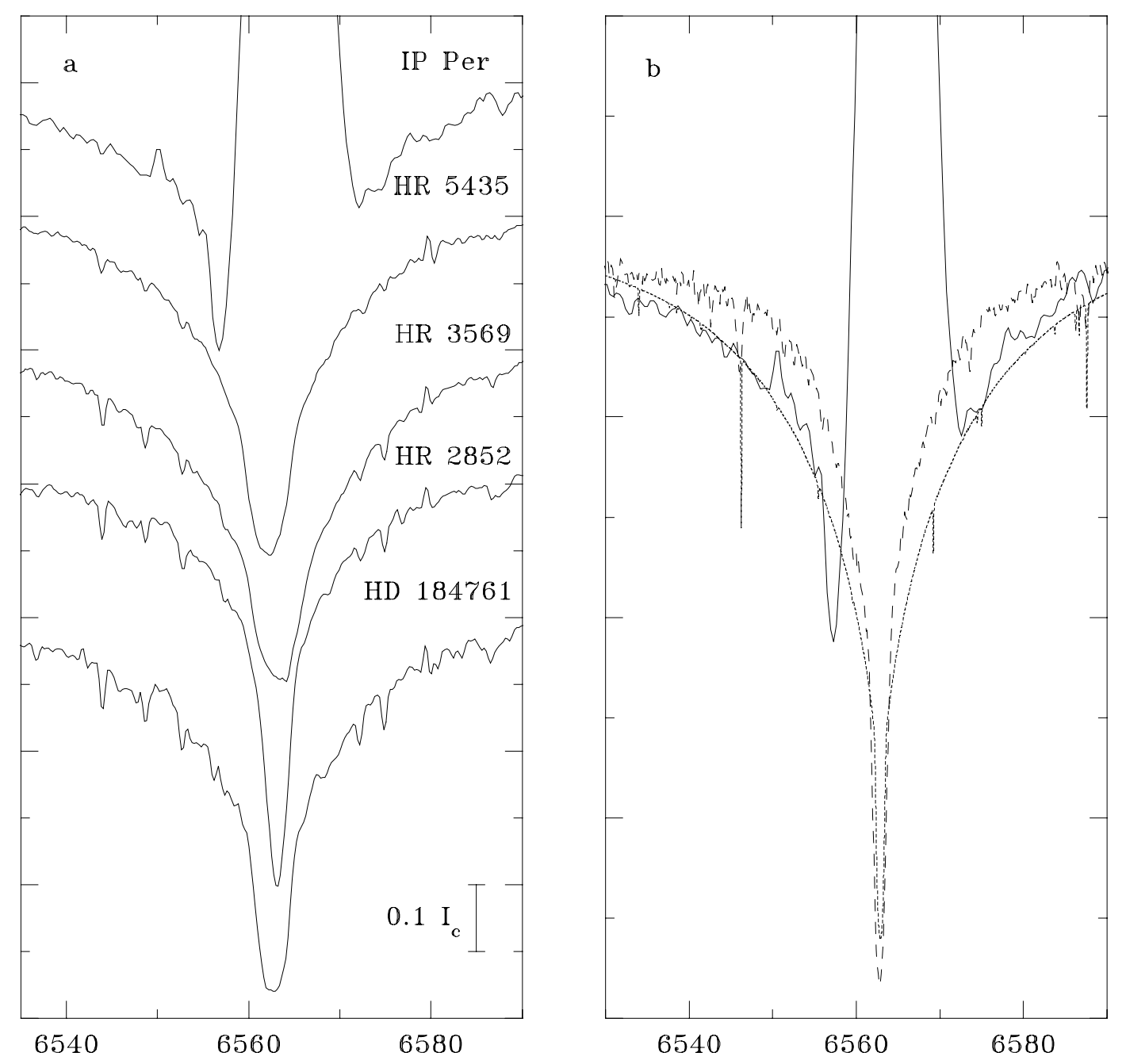

Fig. 5. Comparison of the $\mathrm{H} \alpha$ line wings. a) IP Per and A-type dwarfs. The spectra are shifted by $0.2 I_{\mathrm{c}}$ with respect to each other and marked with the star's names. No correction for telluric lines has been applied. b) IP Per (solid line), HD 59612 (A5 I, dashed line), and our synthetic H $\alpha$ profile (dotted line). The wavelength scale is given in $\AA$.

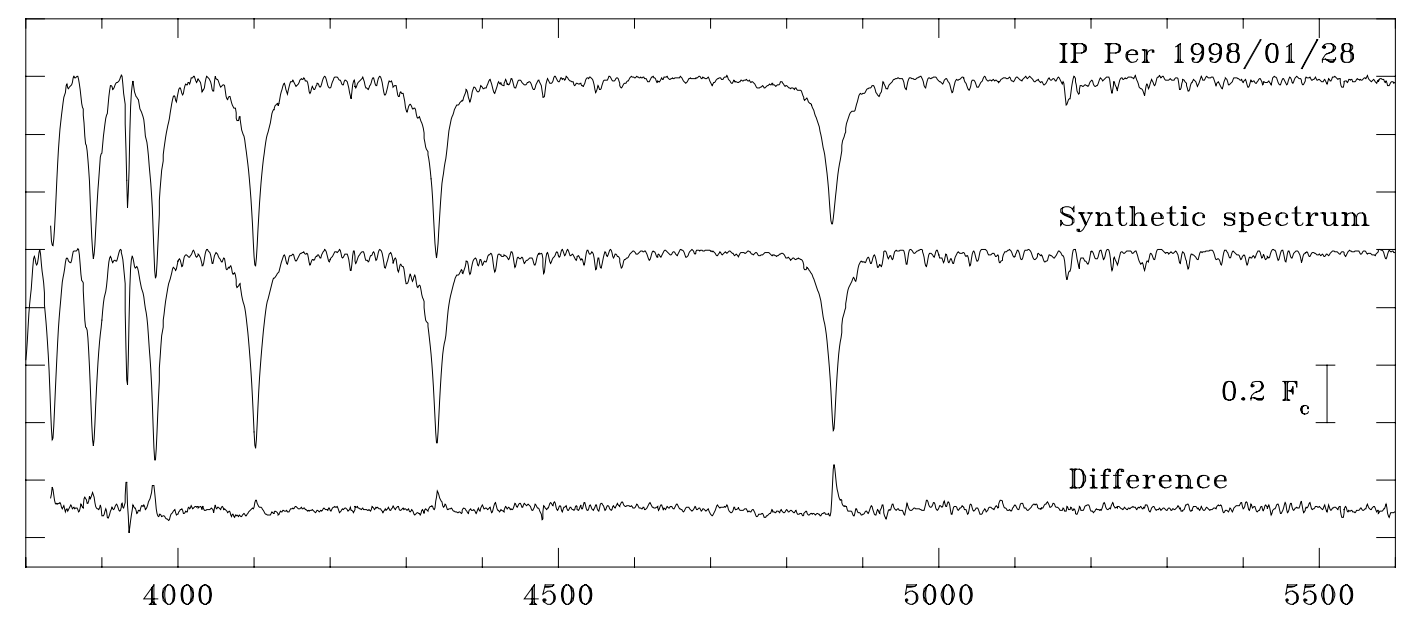

Fig. 6. The model fit to the DSO spectrum of IP Per. At the bottom of the graph is the difference spectrum. The emission in the $\mathrm{H} \beta, \mathrm{H} \gamma$ and $\mathrm{H} \delta$ lines can be seen clearly in the difference spectrum. The structure in the difference spectrum shortwards of $4000 \AA$ is due mostly to a slight wavelength error. All other differences can be attributed to noise. All the spectra are normalized to the continuum flux $\left(F_{\mathrm{c}}\right)$ and shifted with respect to each other. The wavelength scale is given in $\AA$. 
obtained using an échelle spectrograph at an intermediate resolution ( $R \simeq 6000$ ), with the theoretical profiles for $T_{\text {eff }}=8800 \mathrm{~K}$ and $\log g=2.0$. We should note that the discrepancy in $\log g$ is found for the échelle spectra only. Based on the difficulties in the $\mathrm{H} \beta$ profile normalization described here, this discrepancy may have an instrumental origin in part. It seems to have a smaller effect on the $\mathrm{H} \alpha$ profiles, because even the Ritter data with the échelle order width of $70 \AA$ show good agreement with both theoretical and expected gravity values for stars in this temperature region. Additionally, Kovalchuk \& Pugach (1997) assumed a higher $T_{\text {eff }}$, which gives stronger Balmer line profiles at the main-sequence gravity. As a result, they had to lower the gravity in order to match the observed profiles. This effect may be as important as the échelle reduction uncertainties.

In order to study possible systematic effects in the gravity determination, we estimated fundamental parameters of the standard stars used by Kovalchuk \& Pugach (1997) on the basis of the photometric data and HIPPARCOS parallaxes. The results presented in Table 4 show that for 3 stars out of the four our values of $\log g$ are larger by $\sim 0.5$ than those determined by Kovalchuk \& Pugach (1997). The fourth star, HD 32642, is a close binary, which may affect its $\log g$ derived using both ways: from the line profile fitting and from other data for the star. This correction is rather small and cannot explain the remaining difference of $\sim 1.0$ in $\log g$ between the échelle and non-échelle determinations. In any case, there is no physical reason for a star to display different atmospheric parameters in different lines of the same series. In order to reconcile this problem, one can assume that circumstellar emission is observed up to $\pm 1000-1500 \mathrm{~km} \mathrm{~s}^{-1}$ in the wings of $\mathrm{H} \beta$ and higher members of the Balmer series. However, in this case even a stronger emission has to be seen in $\mathrm{H} \alpha$ which is not the case. Therefore, one can probably seek the solution to this problem in data acquisition and reduction. Additionally, the $T_{\text {eff }}-$ gravity interplay may produce multiple solutions in the observed profile fitting. This problem emphasizes the role of careful independent estimates of the fundamental parameters.

\section{Discussion}

The photometric behaviour of IP Per and the presence of a large IR excess are consistent with its classification as a pre-main-sequence Herbig Ae star and a member of the UXOr group. Our spectroscopic results favor its MK classification as an A7 ve star. Using all the information we collected here, we can put independent constraints on the star's fundamental parameters.

The presence of DIBs in the spectrum of IP Per indicate that its colour-indices are affected by interstellar reddening. A small colour-excess of $E_{B-V}=0.15 \pm 0.05 \mathrm{mag}$ is deduced from averaged colour-indices at maximum brightness, consistent with the faint strength of the DIBs detected in the optical (high-resolution) spectrum. This leads to two different estimates of the star's MK type as $\mathrm{A} 6 \pm 1 \mathrm{~V}$ or $\mathrm{A} 8 \pm 1$ I-II which are separated by a large gap in luminosity. The radial velocity $(R V)$ information from our SAO spectrum helps to constrain the luminosity of IP Per. The mean $R V$ of the photospheric absorption lines is $+13 \pm 1 \mathrm{kms}^{-1}$, while the interstellar $\mathrm{Na} \mathrm{I}_{1,2}$ lines have $R V+16 \pm 1 \mathrm{kms}^{-1}$. These values are very close to those of the nearest bright star o Per and stars from Per OB2 association. They suggest a distance of about $300 \mathrm{pc}$, based on the recent determinations for Per OB2 (296 \pm 17 pc, de Zeeuw et al. 1999) and for a nearby cluster IC $348\left(261_{-23}^{+27}\right.$ pc, Scholz et al. 1999). The proper motion of IP Per measured by HIPPARCOS $\left(\mu_{\alpha}=+11.30\right.$ mas yr $^{-1}, \mu_{\delta}=-8.90$ mas yr $^{-1}$, ESA 1997) is within the range of those adopted for the Per OB2 members (see Klochkova \& Kopylov 1985). The interstellar reddening derived here for IP Per agrees with the interstellar extinction law in the direction of Per OB2 (Černis 1993), if its distance is close to $300 \mathrm{pc}$.

On the other hand, $D=300 \mathrm{pc}$ is a lower limit for the distance towards IP Per which is calculated, assuming that its luminosity is not lower than that of the zero-age mainsequence $(\mathrm{ZAMS})$ for its $T_{\text {eff }}\left(\log L_{\mathrm{bol}} / L_{\odot}=1.0\right)$. With these parameters the star would have a mass of $1.8 M_{\odot}$ (Palla \& Stahler 1993) and a gravity $\log g=4.35$. The latter coincides with our result for the star's gravity supporting the $D=300 \mathrm{pc}$ estimate.

The kinematical parameters of IP Per do not favor the high-luminosity case. According to the galactic rotation curve (Dubath et al. 1988), a star in this direction would have a $R V$ of $-(5-10) \mathrm{km} \mathrm{s}^{-1}$ at a distance of $1 \mathrm{kpc}$ and farther. Additionally, at $D=1 \mathrm{kpc}$ IP Per would have a luminosity corresponding to that of the theoretical birthline for intermediate-mass stars (Palla \& Stahler 1993). Such a location would imply that the star is extremely young and surrounded by a large amount of protostellar matter, which usually produces a strong obscuration in the visual region. This is not observed and rules out the extreme youth of IP Per. Furthermore, if we assume that IP Per is an evolved star, then even at $D=3 \mathrm{kpc}$ it would have $\log g \sim 3.0$. However, such a large distance contradicts the observed $R V$ and a rather large proper motion of the star.

Since IP Per displays emission lines and a strong IR excess, its absorption-line spectrum might be affected by circumstellar emission, known as veiling. Indeed, veiling from a possible accretion disk could be present in Herbig $\mathrm{Ae} / \mathrm{Be}$ stars and might affect determination of their fundamental parameters. Böhm \& Catala (1993) approached this problem in their study of a hotter (A0) and more massive star, AB Aur, and estimated an upper limit of the amount of veiling as $3 \%$ at $4500 \AA$ and $\leq 16 \%$ at $6100 \AA$. These authors considered the radiation from a flat accretion disk to be the main source of the veiling. Below we list the reasons justifying that veiling is not important for IP Per.

1. Veiling affects spectral line wings and makes the lines narrower. Therefore, subtraction of any additional 

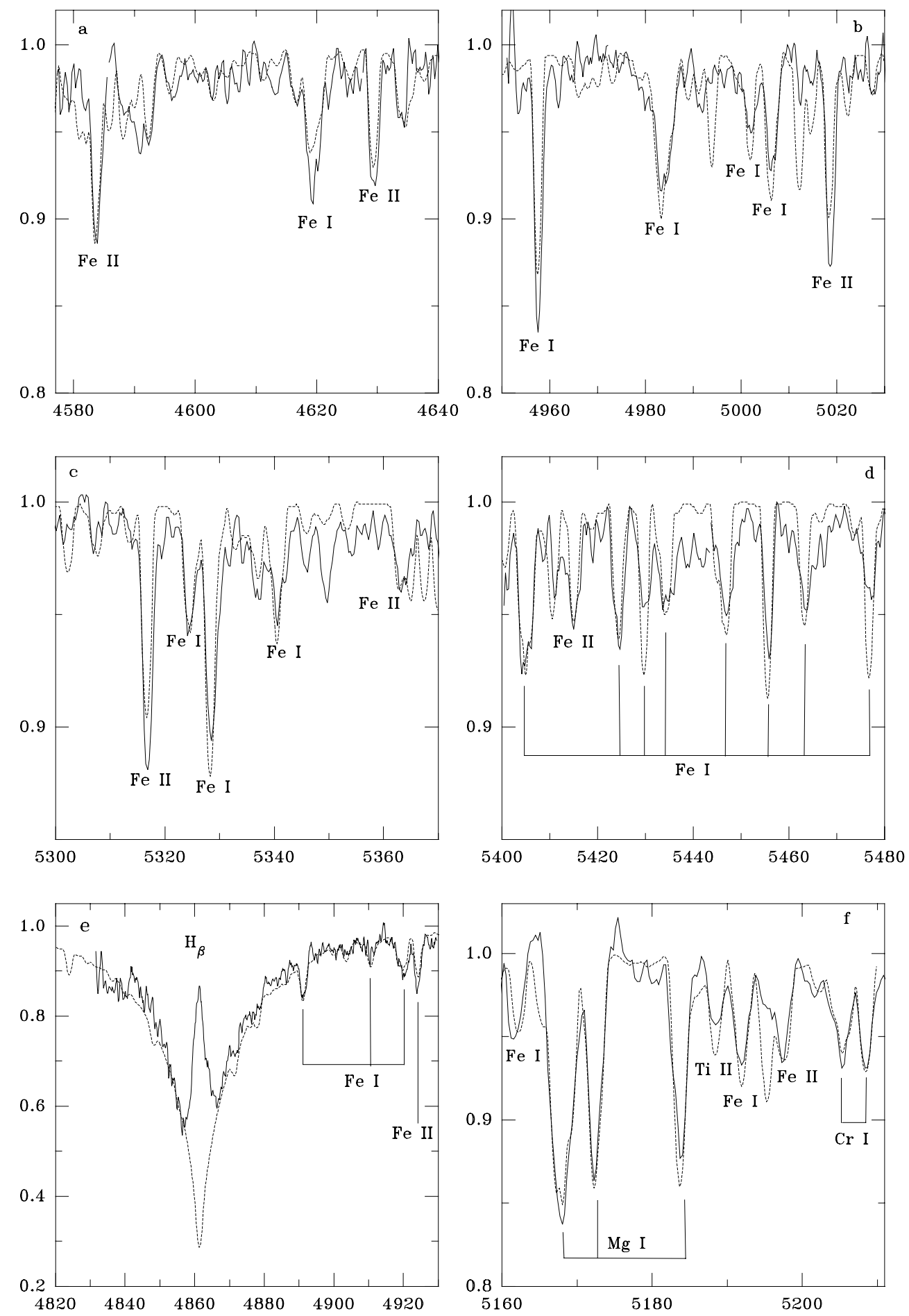

Fig. 7. Comparison of the SAO spectrum of IP Per and our synthetic spectrum. The star's spectrum is shown by solid lines, while the theoretical spectrum for $T_{\text {eff }}=7960 \mathrm{~K}$ and $\log g=4.38$ by dashed lines. The intensities are normalized to the underlying continuum, while the wavelengths are given in $\AA$.

continuum from the observed spectrum would result in higher values for the surface gravity. From our modelling, we already have $\log g=4.4$, which is comparable to those of main-sequence stars.

2. According to current models (see Böhm \& Catala 1993), veiling is wavelength dependent. Thus, the presence of a noticeable amount of it would give us different fundamental parameters for the star from blue and red lines. Figures 5-7 show that there is no such problem for IP Per. Veiling would also change the Balmer jump, which is in agreement with the theoretical spectrum (see Fig. 2). The latter fits both the 
Table 4. Fundamental parameters of the standard stars from Kovalchuk \& Pugach (1997).

\begin{tabular}{rlrrrrrrrrr}
\hline HD & Sp.T. & $V$ & $B-V$ & $U-B$ & $D, \mathrm{pc}$ & $M_{V}$ & Mass, $M_{\odot}$ & $T_{\text {eff }}$ & $\log g$ & $\log g^{a}$ \\
\hline 17 & A2 & 6.89 & 0.15 & 0.10 & $97 \pm 8$ & 1.96 & 2.0 & 8400 & 4.29 & 4.10 \\
23194 & A5 V & 8.07 & 0.20 & 0.15 & 125 & 2.59 & 1.7 & 7800 & 4.35 & 3.65 \\
$32642^{b}$ & A5m & $6.50:$ & 0.21 & 0.22 & $142 \pm 30$ & $0.74:$ & $2.4:$ & 7800 & $3.75:$ & 3.70 \\
82523 & A3 V & 6.52 & 0.12 & 0.09 & $92 \pm 8$ & 1.70 & 2.1 & 8600 & 4.25 & 3.65 \\
\hline
\end{tabular}

The photometric data are taken from Kornilov et al. (1991); distances are calculated from the parallaxes obtained by HIPPARCOS (the mean distance for Pleiades is used for HD 23194, whose parallax was not measured); $T_{\text {eff }}$ are estimated using the optical colour-indices on the basis of the surface brightness method described by Gubotchkin \& Miroshnichenko (1991); while the masses are estimated on the basis of the evolutionary tracks from Schaerer et al. (1993).

${ }^{a}$ Values of $\log g$ listed in the last column are from Kovalchuk \& Pugach (1997).

${ }^{b}$ HD 32642 has a close companion at $1^{\prime \prime} 1$, and all its parameters are uncertain.

jump and line intensities fairly well, suggesting that no significant veiling is present.

3. IP Per is a less massive object than AB Aur, and its evolutionary time in the pre-main-sequence stage is much larger than that of AB Aur. Since IP Per seems to be very close to the zero-age main-sequence, one might suggest that it is also older than AB Aur, and the amount of protostellar matter around it is smaller than that for AB Aur. This, in turn, suggests that the amount of veiling, which is already small for AB Aur, is even smaller for IP Per.

4. Finally, in order to calculate the veiling spectrum, one must introduce a number of accretion disk parameters, which could be constrained using images at different wavelengths (see Miroshnichenko et al. 1999b) in addition to the SED data. However, such images have not been obtained for IP Per yet. Moreover, the above arguments suggest that the amount of veiling is small for IP Per, and thus should have little effect on our results.

\section{Conclusions}

Summarizing all our findings, we suggest that IP Per represents a typical UXOri-type pre-main-sequence star. The analysis of its high- and low-resolution optical spectra strongly favors high gravity $(\log g \simeq 4.4)$ rather than a low one. The metallicity of the object's atmosphere is nearly 40 per cent that of the Sun. The spectral type of the star (A7) is determined on the basis of both photometric and spectroscopic criteria. The star's radial velocity and proper motion suggest that it most likely belongs to the Per OB2 association ( $D \simeq 300$ pc, de Zeeuw et al. 1999). The fundamental parameters we derived here are consistent with the same value for the distance. At $D=300 \mathrm{pc}$, IP Per would be located on the ZAMS with $\log L_{\mathrm{bol}} / L_{\odot}=1.0$ and a mass of $1.8 M_{\odot}$.

Our spectroscopic data show that the most probable value of $\log g$ for IP Per is nearly 4.4. Thus, we do not confirm the finding of Kovalchuk \& Pugach (1997) that IP Per is an evolved object. Morevover, the gravity values of the UXOR group obtained by these authors need to be re-estimated on the basis of a more careful spectroscopic data analysis.

The star displays a noticeably variable emission component in the Balmer lines $(\mathrm{H} \beta-\mathrm{H} 9)$. Unfortunately, this process has not yet been observed in the $\mathrm{H} \alpha$ line, where this emission is the strongest. Follow-up high-resolution data would be very useful to constrain the gaseous envelope parameters, a problem that was not adressed in this paper nor in the previous studies of IP Per. Our nearIR photometry shows that the star's brightness is significantly variable in this region $(\Delta K \sim 0.4 \mathrm{mag})$. This cannot be explained by Algol-type minima and requires further investigation. Far-IR and submillimetric observations are necessary to constrain parameters of the object's dusty environments.

Acknowledgements. We thank E. Verdugo for providing us with the spectrum of HD 59612. A. M. and K. S. B. acknowledge support from NASA grant NAG5-8054 and thank the IRTF staff for their assistance during the observations. Karen Bjorkman is a Cottrell Scholar of the Research Corporation, and gratefully acknowledges their support. R. O. Gray acknowledges the partial support of a Research Corporation Grant. P. G.-L. acknowledges support from grant PB97-1435-C02-02 from the Spanish Dirección General de Enseñanza Superior e Investigación Científica (DGESIC). V. K. acknowledges support from grant 99-0218339 of the Russian Foundation for Basic Research. K. S. B., A. M., and V. K. acknowledge support from the U.S. CRDF, award number RP1-2264. Support for observational research at Ritter Observatory is provided by The University of Toledo and by NSF grant AST-9024802 to B. W. Bopp. Technical support at Ritter is provided by R. J. Burmeister. This publication makes use of data products from the Two Micron All Sky Survey, which is a joint project of the University of Massaachusetts and the Infrared Processing and Analysis Center, funded by the National Aeronautic and Space Administration and the National Science Foundation. 
Appendix. Lines identified in the SAO spectrum of IP Per.

\begin{tabular}{|c|c|c|c|c|c|c|c|c|c|c|c|}
\hline Line ID & $\lambda_{\text {lab }}$ & $I / I_{\mathrm{c}}$ & $E W$ & $R V$ & Rem. & Line ID & $\lambda_{\text {lab }}$ & $I / I_{\mathrm{c}}$ & $E W$ & $R V$ & Rem. \\
\hline Tiнi(81) & 4571.97 & 0.93 & & $15:$ & & FeI $(553)$ & 5324.19 & 0.94 & 0.09 & 15: & \\
\hline Tiпr(39) & 4583.41 & & & & $+\mathrm{f}$ & FeI (15) & 5328.04 & & & & \\
\hline FeII(38) & 4583.83 & 0.91 & 0.17 & $20:$ & & FeI (37) & 5328.53 & 0.90 & 0.18 & 14: & $+\mathrm{p}$ \\
\hline FeI (409) & 4618.77 & 0.93 & 0.13 & 17: & & CrI (18) & 5345.86 & 0.98 & 0.04 & 10: & \\
\hline FeII(37) & 4629.34 & 0.93 & 0.13 & 10 & & FeII(48) & 5362.86 & 0.96 & 0.09 & 9: & \\
\hline CrI (21) & 4652.16 & 0.96 : & & $12:$ & & FeI (15) & 5371.49 & 0.95 & 0.12 & 12: & \\
\hline FeI $(822)$ & 4667.46 & 0.95 & 0.09 & & & Tiпi(69) & 5381.02 & 0.95 & 0.17 & & \\
\hline MgI (11) & 4702.98 & 0.91 & 0.14 & $12:$ & & FeI (15) & 5397.13 & & & & \\
\hline NiI (98) & 4714.42 & 0.96 : & 0.07 & & & FeI (1145) & 5404.14 & & 0.19 & & \\
\hline MnI (21) & 4762.5: & & & & & CrII(23) & 5407.61 & & & & \\
\hline Tini(17) & 4762.78 & 0.96 & 0.07 & 18 & $+\mathrm{p}$ & FeII(48) & 5414.07 & & & & \\
\hline MnI (21) & 4765.86 & & & & & FeI (1165) & 5415.20 & 0.96 & 0.10 & 11: & $+\mathrm{p}$ \\
\hline MnI (21) & 4766.42 & 0.98 & 0.04 & $12:$ & $+\mathrm{p}$ & FeI (1146) & 5424.07 & 0.95 & 0.16 & 18: & \\
\hline FeI $(67)$ & 4771.70 & 0.95 & 0.11 & 14: & & FeI $(15)$ & 5429.7 & 0.97 & 0.07 & 15: & \\
\hline CrII(30) & 4824.13 & 0.94 & 0.12 & & & FeI (15) & 5434.53 & 0.97 & & & \\
\hline $\mathrm{H} \beta$ & 4861.33 & & 2.0 & $5:$ & emis. & FeI (15) & 5446.92 & 0.95 & 0.17 & 8: & \\
\hline FeI (318) & 4871.5: & & 0.08: & 13: & & FeI (15) & 5455.61 & 0.92 & 0.12 & 13: & \\
\hline $\operatorname{CrII}(30)$ & 4876.40 & & 0.15 : & & $+2 \mathrm{f}$ & FeI (1163) & 5463.28 & 0.96 & 0.14 & & \\
\hline CaI (35) & 4878.2: & & & & & FeI $(1029,1062)$ & 5476.8: & 0.95 & 0.12 & & \\
\hline FeI (318) & & & & & & MgI (9) & 5528.39 & 0.95 & $0.24:$ & & \\
\hline FeI (318) & 4891.2: & 0.90 & 0.17 & $12:$ & & FeII(55) & 5534.86 & 0.96 & 0.07 & 15: & \\
\hline FeI () & 4910: & & & & & FeI $(686)$ & 5572.84 & 0.96 & 0.07 & $7:$ & \\
\hline $\operatorname{TiII}(114)$ & 4911.19 & 0.97 & 0.04: & & $+\mathrm{p}$ & FeI $(686)$ & 5586.76 & & & & \\
\hline FeI (318) & 4919.00 & & & & $+\mathrm{f}$ & CaI (21) & 5588.76 & 0.96 & 0.19 & & $+\mathrm{p}$ \\
\hline FeI $(318)$ & 4920.51 & 0.92 & 0.20 & $10:$ & & CaI $(21)$ & 5594.47 & & & & \\
\hline $\operatorname{FeII}(42)$ & 4923.92 & 0.90 & 0.20 & $14:$ & & FeI $(1182)$ & 5594.65 & 0.96 & 0.08 & 16: & $+\mathrm{p}$ \\
\hline FeI (1065) & 4933.34 & & & & & FeI (1182) & 5598.3: & & & & \\
\hline BaI (1) & 4934.08 & 0.86 & 0.26 & & $+\mathrm{p}$ & CaI (21) & 5598.49 & 0.96 & 0.09 & 10: & $+\mathrm{p}$ \\
\hline FeI $(318)$ & 4957.30 & 0.86 & 0.27 & $14:$ & & FeI $(209)$ & 5615.3 & & & & \\
\hline TiI (71) & 4981.3 & & & & & FeI $(686)$ & 5615.65 & 0.95 & 0.13 & 12: & $+\mathrm{p}$ \\
\hline FeI (1066) & 4983.26 & 0.92 & 0.22 & & $+\mathrm{p}$ & $\operatorname{ScII}(29)$ & 5657.87 & & & & \\
\hline FeI (318) & 4985.50 & 0.92 & & & & $\operatorname{ScII}(29)$ & 5658.34 & & & & \\
\hline FeI (1065) & 4991.27 & 0.97 & & 10: & & FeI $(686)$ & 5658.83 & 0.95 & 0.25 & & $+2 \mathrm{p}$ \\
\hline FeI $(965)$ & 5001.9: & & & & & DIB & 5780 & $0.11:$ & $0.11:$ & & \\
\hline FeI (687) & 5002.79 & 0.96 & 0.07 & & $+\mathrm{p}$ & DIB & 5797 & $\leq 0.02$ & $\leq 0.02$ & & \\
\hline FeI (318) & 5006.13 & 0.94 & 0.12 & $16:$ & & HeI (11) & 5875.63 & 1.02 & 0.21 & & emis. \\
\hline $\operatorname{FeII}(42)$ & 5018.43 & 0.88 & 0.23 & 15 & & NaI (1) & 5889.95 & 0.57 & 0.45 & $20:^{a}, 16^{b}$ & \\
\hline FeI (1094) & 5040.90 & & & & & NaI (1) & 5895.92 & 0.58 & 0.41 & $20:^{a}, 16^{b}$ & \\
\hline
\end{tabular}

Comments on the line list in the spectrum of IP Per.

Unreliable identifications and measurements are denoted by colons.

Intensities at line peaks in units of the underlying continuum are given in Cols. 3 and 9,

$E W$ in $\AA$ in Cols. 4 and 10, heliocentric $R V$ in $\mathrm{km} \mathrm{s}^{-1}$ in Cols. 5 and 11 .

${ }^{a} R V$ of the stellar component; ${ }^{b} R V$ of the interstellar component.

Equivalent widths of only emission parts of $\mathrm{H} \alpha$ and $\mathrm{H} \beta$ are listed.

"p" refers to the preceding line, while "f" to the following one. 
Appendix. continued.

\begin{tabular}{|c|c|c|c|c|c|c|c|c|c|c|c|}
\hline Line ID & $\lambda_{\text {lab }}$ & $I / I_{\mathrm{c}}$ & $E W$ & $R V$ & Rem. & Line ID & $\lambda_{\text {lab }}$ & $I / I_{\mathrm{c}}$ & $E W$ & $R V$ & Rem. \\
\hline $\operatorname{SiII}(5)$ & 5041.03 & & & & & $\operatorname{BaII}(2)$ & 6141.72 & 0.96 & 0.06 & 7 & \\
\hline FeI $(36)$ & 5041.76 & 0.93 & 0.10 & 10: & $+2 \mathrm{p}$ & FeII(74) & 6147.74 & 0.98 & 0.08 & & \\
\hline FeI(1092) & 5097.00 & 0.97 & 0.13 & & & FeII(74) & 6149.25 & & & & \\
\hline NiI $(141,161)$ & 5099.5: & & & & & Oi (10) & 6156.0 & & & & \\
\hline FeI $(16,36)$ & 5107.5 & 0.97 & 0.04 & & & OI (10) & 6156.8 & & & & \\
\hline FeI (1090) & 5125.12 & & & & $+\mathrm{f}$ & Oi (10) & 6158.18 & 0.98 & & & $+2 \mathrm{p}$ \\
\hline NiI (160) & 5125.23 & 0.98 & 0.08 & 13: & & CaI (3) & 6162.17 & 0.96 & 0.06 & $15:$ & \\
\hline FeI (1092) & 5133.69 & 0.97 & 0.05 & $17:$ & & FeII(74) & 6238.39 & & & & \\
\hline FeI $(383)$ & 5139.26 & 0.95 & 0.14 & $12:$ & & FeII(74) & 6239.94 & $0.97:$ & & & $+\mathrm{p}$ \\
\hline FeI $(1092,16)$ & & & & & $+\mathrm{f}$ & $\operatorname{ScII}(28)$ & 6245.62 & & & & \\
\hline NiI (161) & 5142.8: & 0.96 & 0.06 & 10: & & FeI $(816)$ & 6246.32 & & & & \\
\hline FeI $(1090,1095)$ & 5148.17: & 0.97 & 0.05 & & & $\operatorname{FeII}(74)$ & 6247.54 & $0.96:$ & & 5: & $+2 \mathrm{p}$ \\
\hline $\operatorname{CrII}(24)$ & 5153.49 & & & & $+\mathrm{f}$ & DIB & 6278 & 0.91 & 0.27 : & & $+\mathrm{f}$ \\
\hline $\operatorname{Tin}(70)$ & 5154.07 & 0.96 & 0.07 & 12: & & DIB & 6283 & & & & \\
\hline FeI (1089) & 5162.29 & 0.94 & 0.13 & & & OI $(1)$ & 6300.23 & 0.05 & 0.05 & 30 & emis. \\
\hline MgI (2) & 5167.32 & & & & & FeII () & 6317.99 & & & & \\
\hline FeII(42) & 5169.03 & 0.82 & 0.50 & 10: & $+\mathrm{p}$ & FeI(168) & 6318.02 & 0.96 & 0.09 & & $+\mathrm{p}$ \\
\hline MgI (2) & 5172.68 & 0.87 & 0.26 & 14: & & $\operatorname{Sin}(2)$ & 6347.09 & 0.92 & 0.15 & 10 & \\
\hline MgI (2) & 5183.60 & 0.80 & 0.24 & 15: & & FeII(74) & 6369.47 & & & & \\
\hline $\operatorname{Tini}(70)$ & 5188.68 & & & & $+\mathrm{f}$ & $\operatorname{SiII}(2)$ & 6371.36 & 0.95 & 0.08 & 13: & \\
\hline CaI (49) & 5188.84 & 0.96 & & & & FeII(74) & 6416.91 & & 0.07 & & \\
\hline FeI $(383)$ & 5191.46 & & & & & FeI $(62)$ & 6430.85 & & & & \\
\hline FeI $(383)$ & 5192.35 & 0.93 & 0.12 & $12:$ & $+\mathrm{p}$ & FeII(40) & 6432.70 & 0.94 & 0.08 & & $+\mathrm{p}$ \\
\hline FeII(49) & 5197.57 & 0.94 & 0.10 & 6: & & CaI (18) & 6439.08 & 0.90 & & & \\
\hline CrI (7) & 5204.52 & & & & $+\mathrm{f}$ & CaI (19) & 6455.6 & & & & \\
\hline CrI (7) & 5206.04 & 0.95 & 0.11 & & & $\operatorname{FeII}(74)$ & 6456.38 & 0.95 & 0.08 & $17:$ & $+\mathrm{p}$ \\
\hline CrI (7) & 5208.44 & & & & & CaI (18) & 6493.78 & & & & \\
\hline FeI $(553)$ & 5208.59 & 0.94 & 0.10 & 13: & $+\mathrm{p}$ & FeI (1258) & 6496.47 & & & & \\
\hline FeI (36): & 5216.31 & 0.95 & 0.11 & & $+\mathrm{f}$ & $\operatorname{BaII}(2)$ & 6496.90 & 0.94 & & & $+\mathrm{p}$ \\
\hline FeI (36): & 5217.39 & 0.97 & & & & FeII(40) & 6516.05 & 0.93 & 0.11 & & \\
\hline FeI $(383)$ & 5226.87 & & & & & $\mathrm{H} \alpha$ & 6562.82 & 5.16 & 33.1 & $25:$ & emis. \\
\hline FeI (37) & 5227.19 & 0.87 & 0.26 & $14:$ & $+\mathrm{p}$ & DIB & 6614 & $0.92:$ & 0.07 & & \\
\hline FeI (383) & 5232.95 & 0.94 & & & & C I (26) & 7113.16 & & & & \\
\hline FeII(49) & 5234.62 & 0.90 & 0.28 & $12:$ & $+\mathrm{p}$ & C I (26) & 7115.19 & 0.95 & 0.30 & 10: & $+\mathrm{p}$ \\
\hline FeI $(15)$ & 5269.54 & & & & & FeI (1274) & 7389.4 & 0.92 & 0.23 & 10: & \\
\hline FeI (15) & 5270.35 & 0.88 & 0.22 & $12:$ & $+\mathrm{p}$ & OI (1) & 7771.96 & 0.90 & 0.45 & & $+2 \mathrm{f}$ \\
\hline FeII(49) & 5276.0: & $0.90:$ & 0.16 & $13:$ & & OI $(1)$ & 7771.96 & 0.90 & 0.45 & & $+2 \mathrm{f}$ \\
\hline FeII(49) & 5316.61 & 0.88 & 0.20 & 8: & & OI $(1)$ & 7775.40 & & & & \\
\hline
\end{tabular}

\section{References}

Arribas, S., \& Martínez-Roger, C. 1987, A\&AS, 70, 303

Bergner, Yu. K., Bondarenko, S. L., Miroshnichenko, A. S., et al. 1988, Izvestia Glavn. Astron. Observ. v Pulkove, 205, 142

Böhm, T., \& Catala, C. 1993, A\&AS, 101, 629

Böhm, T., \& Catala, C. 1994, A\&A, 290, 167
Böhm, T., \& Catala, C. 1995, A\&A, 301, 155

Černis, K. 1993, Baltic Astron., 2, 214

Coluzzi, R. 1993, Bull. Inf. CDS, 43, 7

de Zeeuw, T., Hoogerwerf, R., de Bruijne, J. H. J., Brown, A. G. A., \& Blaaw, A. 1999, AJ, 117, 354

Dubath, P., Mayor, M., \& Burki, G. 1988, A\&A, 205, 77

Eimontas, A., \& Sudžius, J. 1998, Baltic Astron., 7, 407

ESA 1997, The Hipparcos and Tycho Catalogues, ESA SP-1200 
Faraggiana, R., Gerbaldi, M., van't Veer, C., \& Floquet, M. 1988, A\&A, 201, 259

Glass, I. S., \& Penston, M. V. 1974, MNRAS, 167, 237

Gray, R. O., \& Garrison, R. F. 1989, ApJS, 70, 623

Gray, R. O. 1998, AJ, 116, 2530

Gray, R. O., \& Corbally, C. J. 1994, AJ, 107, 742

Gray, R. O., \& Corbally, C. J. 1998, AJ, 116, 2530

Grinin, V. P., Kiselev, N. N., Chernova, G. P., Minikulov, N. Kh., \& Voshchinnikov, N. V. 1991, Ap\&SS, 186, 283

Gubotchkin, A. N., \& Miroshnichenko, A. S. 1991, Kinematika i fizika neb. tel, 7, 64

Herbig, G. H. 1960, ApJS, 4, 337

Herbig, G. H., \& Bell, K. R. 1988, Lick Obs. Bull. No. 1111

Hoffmeister, C. 1949, Astron. Nachr. Erg., 12, Heft 1

Ivezić, Ž., \& Elitzur, M. 1995, ApJ, 445, 415

Kardopolov, V. I., \& Phylipjev, G. K. 1982, Variable Stars (Peremennye Zvezdy), 21, 688

Klochkova, V. G., \& Kopylov, I. M. 1985, Bull. Spec. Astrophys. Obs., 20, 3

Kolotilov, E. A., Zaitseva, G. V., \& Shenavrin, V. I. 1977, Afz, 13,253

Kornilov, V. G., et al. 1991, Proc. of the Sternberg Astron. Inst., v.63

Kovalchuk, G. U., \& Pugach, A. F. 1997, A\&A, 325, 1077

Kurucz, R. L. 1979, ApJS, 40, 1

Kurucz, R. L. 1993, CD-ROM No. 13, ATlas9 Stellar Atmosphere Programs and $2 \mathrm{~km} / \mathrm{s}$ Grid (Cambridge: Smithsonian Astrophysical Obs.)

Lastennet, E., Valls-Gabaud, D., Lejeune, T., \& Oblack, E. 1999, A\&A, 349, 485

Meinunger, L. 1967, Mitt. Verand. Stern, 4, 63

Miroshnichenko, A. S., Gray, R. O., Vieira, S. L., Kuratov, K. S., \& Bergner, Yu. K. 1999a, A\&A, 347, 137

Miroshnichenko, A. S., Ivezić, Ž., Vinković, D., \& Elitzur, M. 1999b, ApJ, 520, L115
Miroshnichenko, A. S., Mulliss, C. L., Bjorkman, K. S., et al. 1999c, MNRAS, 302, 612

Natta, A., Grinin, V. P., Mannings, V., \& Ungerechts, H. 1997, ApJ, 491, 885

Palla, F., \& Stahler, S. W. 1993, ApJ, 418, 414

Panchuk, V. E., et al. 1998, Bull. Spec. Astrophys. Obs., 44, 127

Panchuk, V. E., et al. 1999, Preprint Spec. Astrophys. Obs., N139

Pirzkal, N., Spillar, E. J., \& Dyck, H. M. 1997, ApJ, 481, 392

Pugach, A. F. 1996, Variable Stars (Peremennye Zvezdy), 23, 391

Schaerer, D., Charbonnel, C., Meynet, G., Maeder, A., \& Schaller, G. 1993, A\&AS, 102, 339

Scholz, R.-D., et al. 1999, A\&AS, 137, 305

Smalley, B., \& Dworetsky, M. M. 1993, A\&A, 271, 515

Solano, E., \& Fernley, J. 1997, A\&AS, 122, 131

Scrutskie, M. F., Schneider, S. E., Stiening, R., et al. 1997, in Proc. Workshop, The Impact of Large Scale Near-IR Sky Surveys, 25

Straizhis, V. 1977, Multicolour stellar photometry, Vilnius, Mokslas publ.

Straižys, V., Crawford, D. L., \& Philip, A. G. D. 1996, Baltic Astron., 5, 83

Strom, S. E., Strom, K. M., Yost, J., Carrasco, L., \& Grasdalen, G. 1972, ApJ, 173, 353

Testi, L., Palla, F., Prusti, T., Natta, A., \& Maltagliati, S. 1997, A\&A, 320, 159

Thé, P. S., de Winter, D., Peréz, M. R. 1994, A\&AS, 104, 315

Verdugo, E., Talavera, A., \& Gómez de Castro, A. I. 1999, A\&A, 346, 819

Weaver, Wm. B., \& Jones, G. 1992, ApJS, 78, 239

Weintraub, D. A. 1990, ApJS, 74, 575

Wenzel, W. 1978, Mitt. Verand. Stern, 8, 53 